\title{
Digital fabrication of multi-material biomedical objects
}

\author{
H.H. Cheung and S.H. Choi* \\ Department of Industrial and Manufacturing Systems Engineering, \\ The University of Hong Kong, Pokfulam Road, Hong Kong, China.
}

\begin{abstract}
This paper describes a multi-material virtual prototyping (MMVP) system for modelling and digital fabrication of discrete and functionally graded multimaterial objects for biomedical applications. The MMVP system consists of a DMMVP module, an FGMVP module, and a virtual reality (VR) simulation module. The DMMVP module is used to model discrete multi-material (DMM) objects, while the FGMVP module is for functionally graded multi-material (FGM) objects. The VR simulation module integrates these two modules to perform digital fabrication of multi-material objects, which can be subsequently visualized and analyzed in a virtual environment to optimize MMLM processes for fabrication of product prototypes. Using the MMVP system, two biomedical objects, including a DMM human spine and an FGM intervertebral disc spacer are modelled and digitally fabricated for visualization and analysis in a VR environment. These studies show the MMVP system is a practical tool for modelling, visualization, and subsequent fabrication of biomedical objects of discrete and functionally graded multi-materials for biomedical applications. The system may be adapted to control MMLM machines with appropriate hardware for physical fabrication of biomedical objects.
\end{abstract}
Keywords: digital fabrication, virtual prototyping, multi-material, biomedical objects, toolpath planning

\section{Introduction}

Recent developments in medical and dental fields have warranted multi-material objects or implants with desirable properties for biomedical applications. In comparison, a discrete multi-material (DMM) object helps differentiate one part from others, or tissues from blood vessels of a human organ, while an functionally graded multi-material (FGM) object often performs better in rigorous environments. In particular, suitably graded composition transitions across multi-material interfaces can create an object of very different properties to

\footnotetext{
*Correspondence author, email: shchoi@hku.hk
} 
suit various applications [1,2]. For example, dental implants made of FGM structures composed of titanium (Ti) and hydroxyapatite (HAP) can better satisfy both mechanical and biocompatible property requirements [3].

Some researchers have indeed attempted to fabricate such objects, like artificial hip joints, intervertebral disc spacers, tooth implants or tooth crowns, tissue scaffolds, and bone and jaw structures, for studying and planning complex surgical operations, implantation, and as specimens for experiments in pharmaceutical manufacturing enterprises and hospitals. Wang et al. [4] developed a precision extruding deposition (PED) system to fabricate interconnected 3D scaffolds. Kai et al. [5] developed a double-nozzle low-temperature deposition manufacturing technique to fabricate the functional gradient bone repair biomaterials for bone tumour surgery.

Despite these research efforts, fabrication of multi-material objects remains a challenge. Recently, much work has been devoted to adapting Layered Manufacturing (LM) to fabricate multi-material objects. A few experimental multi-material layered manufacturing (MMLM) machines, such as a discrete multiple material selective laser sintering $\left(\mathrm{M}^{2} \mathrm{SLS}\right)$ machine [6,7], a shape deposition manufacturing machine [8,9], a fused deposition of multiple ceramics (FDMC) machine [10], and a 3D inkjet-printing machine [11-13] have been developed.

For biomedical applications, Xu et al. [14] developed a Solid Freeform Fabrication-based Slurry Extrusion system for fabrication of the multi-material dental crown for dental implantations. Bandyopadhyay et al. [15] developed a Laser Engineered Net Shaping $\left(\right.$ LENS $^{\mathrm{TM}}$ ) system to fabricate porous and functionally graded structures for load bearing implants. Kim et al. [16] reported a Nano Composite Deposition (NCD) system for fabrication of functionally graded biomaterials.

Although these systems seemed suitable for relatively simple objects of a limited variety of materials, they provided a good foundation for further hardware development. While the development of MMLM involves both software and hardware issues, it is essential to develop an integrated software system for modelling and fabrication of complex multi-material objects.

To fabricate multi-material prototypes, both material and geometric information must be made available. Although STL is a de-facto industrial standard file format for LM, it only contains geometric information. Therefore, some researchers have proposed CAD representation methods for DMM objects or FGM objects to facilitate general CADCAM applications, including MRPII [17-24]. Some of these representation methods would be useful 
for MMLM when fully developed and widely adopted. However, most proposed methods cannot conveniently represent complex multi-material objects, particularly for biomedical applications.

Most complex biomedical models, such as human organs and bone structures, are not designed using CAD systems. Instead, they are captured by laser digitizers, or CT, MRI, and micro CT scanners. Sun et al. [25] reviewed the use of CT and MRI techniques to model tissue scaffolds as CAD models that can be used for biomimetic design, analysis, simulation, and freeform fabrication of the tissue scaffolds. In general, the digitized images are normally processed to form a model in STL format with no material or topological information needed to extract the slice contours. Indeed, slice contours are random in nature without any explicit topological hierarchy relationship, and to process them for fabrication remains a challenging obstacle that has yet to be surmounted. Most of the above representation schemes cannot conveniently represent objects from CT and MRI images, and subsequent processing of multimaterial objects for fabrication control is ignored. Hence, it is worthwhile to develop an integrated computer system to represent and process multi-material biomedical objects for subsequent generation of toolpaths for fabrication control.

This paper therefore describes a multi-material virtual prototyping (MMVP) system for modelling, visualization, and digital fabrication of discrete and functionally graded multimaterial objects for biomedical applications. The MMVP system offers flexibility in representing such objects from either CAD models or CT/MRI scan images. It also provides a virtual reality (VR) environment for digital fabrication, visualization, and quality analysis of multi-material biomedical prototypes. As such, the need for physical prototyping is minimized, and the cost and time of biomedical product development reduced substantially. Hence, the MMVP system provides a practical tool to represent and visualize biomedical objects for planning surgical operations, such as dental and spine disc implantations. The system may be adapted for control of MMLM machines, when appropriate hardware becomes available, for physical fabrication of multi-material biomedical objects.

\section{The MMVP System}

The MMVP system is an integrated software system for modelling, visualization, and digital fabrication of multi-material objects for engineering and biomedical applications. It consists mainly of (i) a discrete multi-material virtual prototyping (DMMVP) module for DMM objects; (ii) a functionally graded multi-material virtual prototyping (FGMVP) module for functionally graded multi-material objects; and (iii) a virtual prototyping (VP) module that integrates DMMVP and FGMVP with virtual reality (VR) for visualization and optimization 
of MMLM processes for digital fabrication and quality analysis of both discrete and functionally graded multi-material objects.

\subsection{The DMMVP Module}

The DMMVP is a main module of the MMVP system, consisting of a suite of software packages for modelling and visualization of multi-material objects and simulation of MMLM process. These packages include a colour modeller for colouring monochrome STL models, a slicing algorithm, a topological hierarchy-sorting algorithm for grouping random slice contours of DMM objects, a topological hierarchy-based toolpath planning algorithm for generation of sequential and concurrent multi-toolpaths, and a virtual prototyping (VP) module for digital fabrication of DMM objects. It works as follows.

Firstly, a biomedical model created by CAD or an MRI/CT digitizer is converted into STL format. As STL is monochrome or single-material, an in-house package is used to paint the STL model, with each colour representing a specific material.

Secondly, a few steps are undertaken to prepare for subsequent simulation of the MMLM process and visualization of the resulting digital prototypes: (a) slice the colour STL model into a number of layers of a predefined thickness. The resulting layer contours and material information are stored in a modified Common Layer Interface (CLI) file; (b) sort the slice contours with a contour sorting algorithm to establish explicit topological hierarchy; (c) based on the hierarchy information, multi-toolpath planning algorithms are used to plan and generate multi-toolpaths by hatching the slice contours with a predefined hatch space. The hatch vectors are stored in the modified CLI file for prototype fabrication and build-time estimation.

Thirdly, the VP module performs digital fabrication of multi-material prototypes. The resulting prototypes can be stereoscopically visualized and analyzed to review and improve the design of the biomedical objects efficiently.

The following section uses a human dextrocardic heart to demonstrate how the DMMVP module can represent and fabricate multi-material objects for biomedical applications, such as surgical training and planning, patient's education, and implantations.

Dextrocardia is a heart disease in which the primitive heart tube folds to the left in a mirror image of a normal bulboventricular loop. Figure 1 shows a monochrome human dextrocardic heart model in STL format constructed from CT or MRI images. To differentiate various parts 
of the heart, the colour STL modeller is used to paint the model. As shown in figure 2, the colour model helps visualize and differentiate the various parts of the dextrocardic heart more vividly to explain and plan complex surgical operations. Moreover, each colour represents a specific type of material, and hence a colour STL model can provide both geometric and material information for design processing and subsequent fabrication on an MMLM machine.
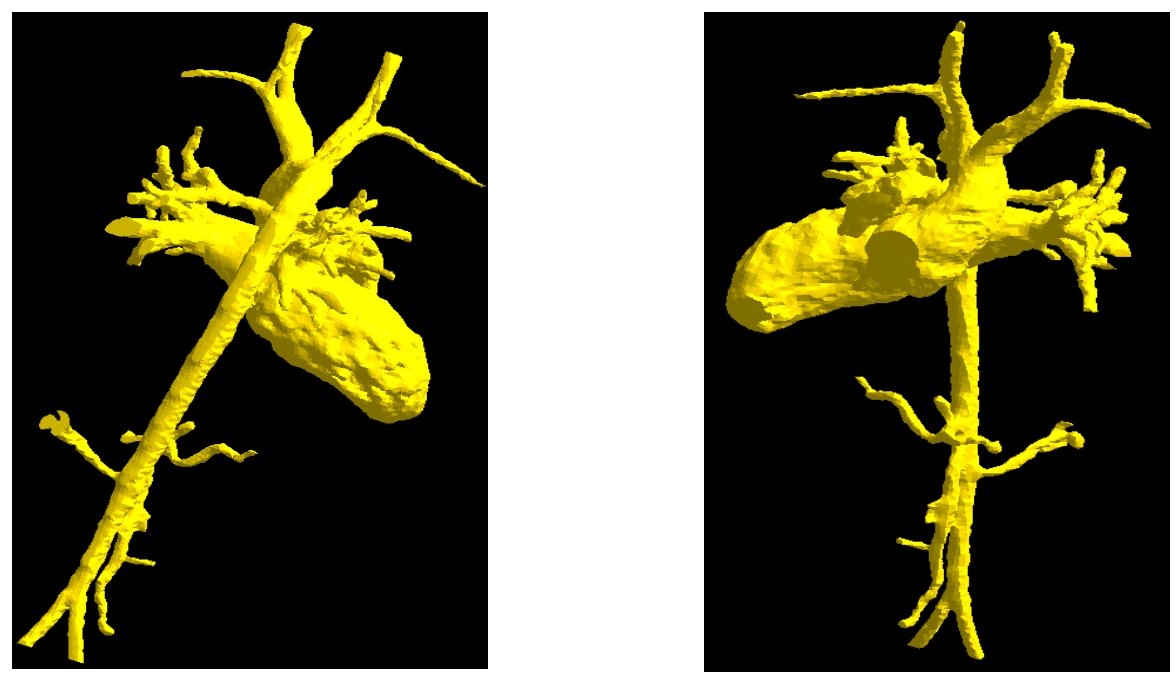

Figure 1. A monochrome STL model of a human dextrocardic heart

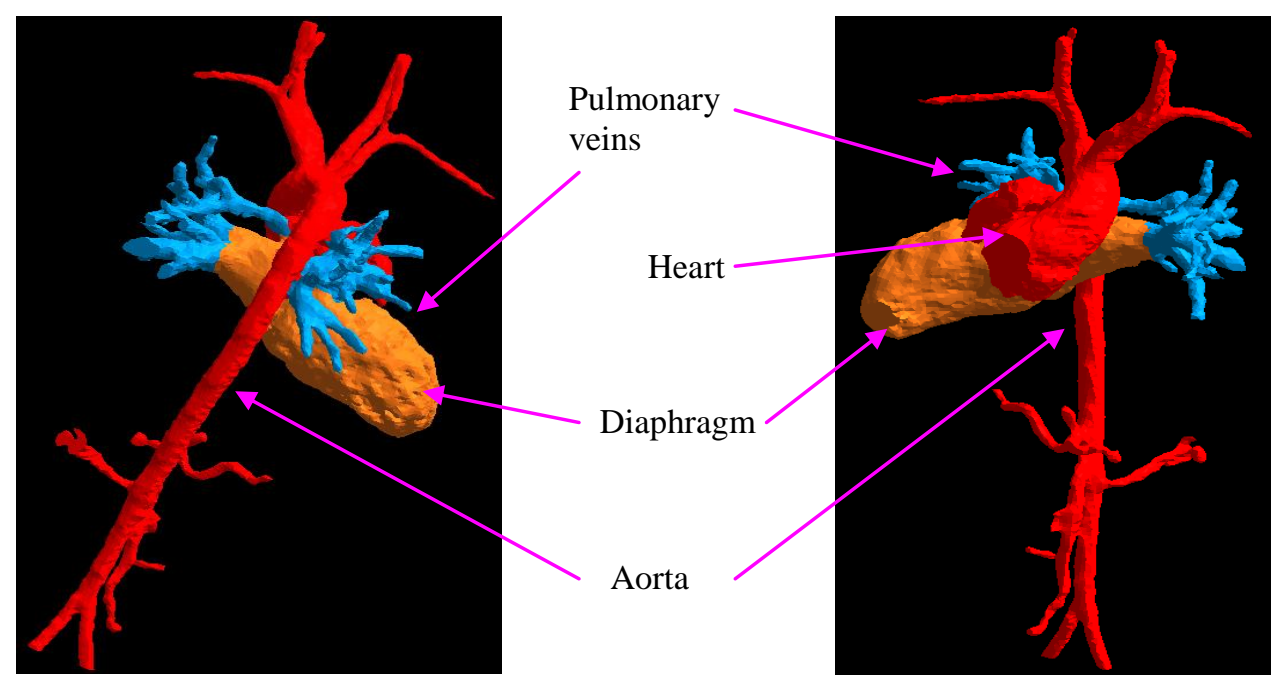

Figure 2. A colour STL model of the human dextrocardic heart

For example, to fabricate this heart prototype with discrete multi-materials, a set of nozzles $\left(\mathrm{N}_{\mathrm{i}}, \mathrm{i}=1,2, \ldots \mathrm{n}\right)$ would deposit specific materials on appropriate slice contours. It is necessary to identify and relate specific contours of a slice to a particular tool and subsequently arrange the toolpaths to fabricate the prototype efficiently. This requires a multitoolpath planning algorithm to generate toolpaths without tool collisions. However, most multi-material objects tend to be complex and the slice contours do not possess any explicit topological hierarchy relationship. As a result, it is very difficult to associate specific contours with a particular tool. To tackle this problem, a topological hierarchy-based approach to 
toolpath planning for MMLM was proposed by the authors [26, 27]. This approach adopts a topological hierarchy-sorting algorithm to construct the topological hierarchy in terms of a parent-and-child list that defines the containment relationship of the contours of a slice. Thus, with the hierarchy relationship, it is no longer necessary to identify and relate contours to a particular nozzle one by one for multi-toolpath planning. Indeed, only grouping of the outermost contours is required. Besides, parametric polygons are used to construct tool envelopes for contour families with the same material property to simplify detection of tool collisions during concurrent movements of nozzles. Hence, concurrent toolpaths without redundant movements and collisions can be easily generated for controlling MMLM machines to fabricate physical multi-material prototypes.

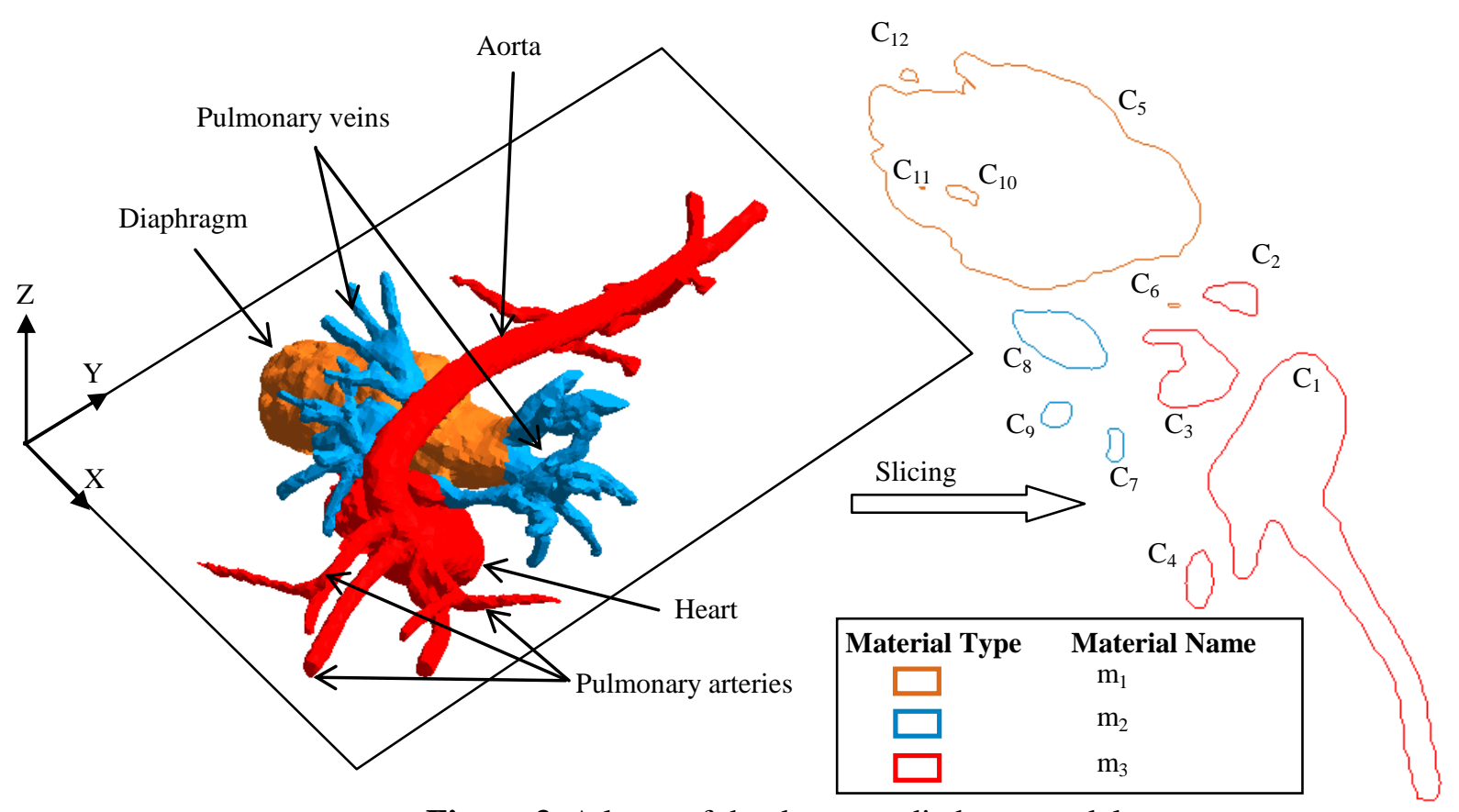

Figure 3. A layer of the dextrocardic heart model

A layer containing 12 contours, as shown in figure 3 , is to be made of three kinds of materials, namely $\mathrm{m}_{1}, \mathrm{~m}_{2}$, and $\mathrm{m}_{3}$. Three nozzles, $\mathrm{N}_{1}, \mathrm{~N}_{2}$, and $\mathrm{N}_{3}$, are allocated to deposit specific materials, $\mathrm{m}_{1}, \mathrm{~m}_{2}$, and $\mathrm{m}_{3}$, respectively. Figure 4 shows the topological hierarchy relationship of the contours in figure 3. According to the contour families, ten toolpaths namely, $\mathrm{P}_{\mathrm{C} 1}, \mathrm{P}_{\mathrm{C} 2}$, $\mathrm{P}_{\mathrm{C} 3}, \mathrm{P}_{\mathrm{C} 4}, \mathrm{P}_{\mathrm{C} 5,10,11}, \mathrm{P}_{\mathrm{C} 6}, \mathrm{P}_{\mathrm{C} 7}, \mathrm{P}_{\mathrm{C} 8}, \mathrm{P}_{\mathrm{C} 9}$, and $\mathrm{P}_{\mathrm{C} 12}$ are generated. Subsequently, the toolpaths with the same material properties are grouped together to form three toolpath-sets, namely $S_{1}$ to $S_{3}$, which are associated with three nozzles from $\mathrm{N}_{1}$ to $\mathrm{N}_{3}$, respectively. Three work envelopes from $E_{1}$ to $E_{3}$ for each of these nozzles are constructed to facilitate planning of concurrent multi-toothpaths. Hence, using the hierarchy information and association relationship between the toolpath-sets and the nozzles, concurrent toolpaths without redundant tool movements and collisions can be easily generated and planned for fabrication control. With the toolpaths, the 
heart prototype can now be digitally fabricated using the VP module [28, 29]. Figure 5 shows the digital fabrication process of a few layers of the heart.

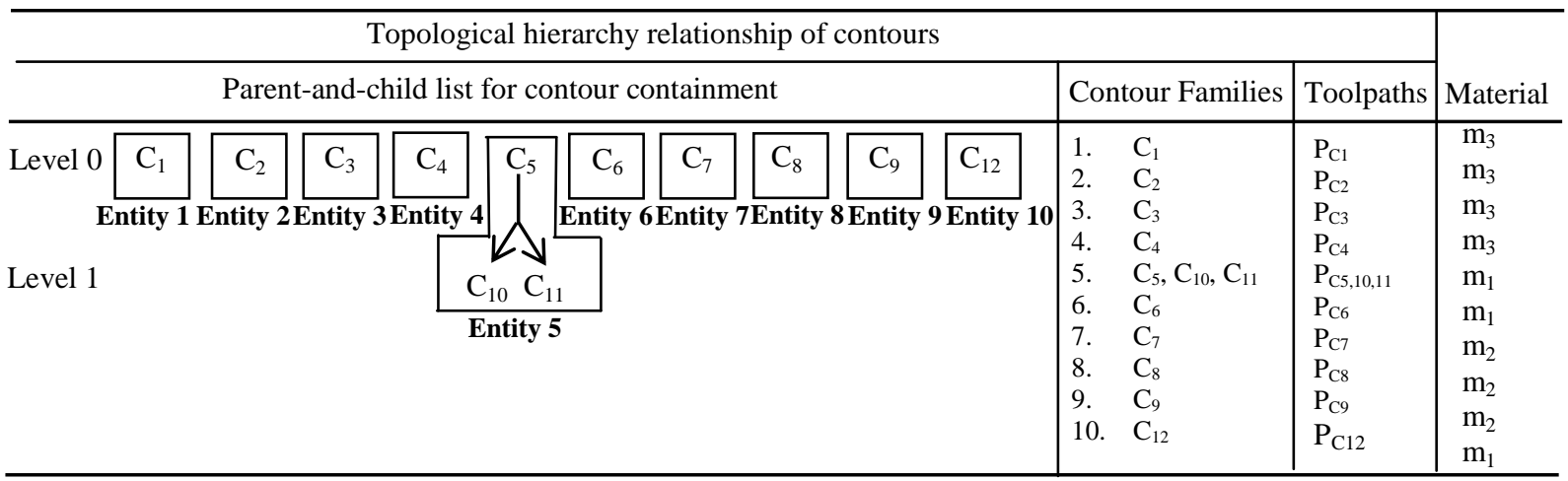

Figure 4. Topological hierarchy relationship of the heart layer in Fig. 3

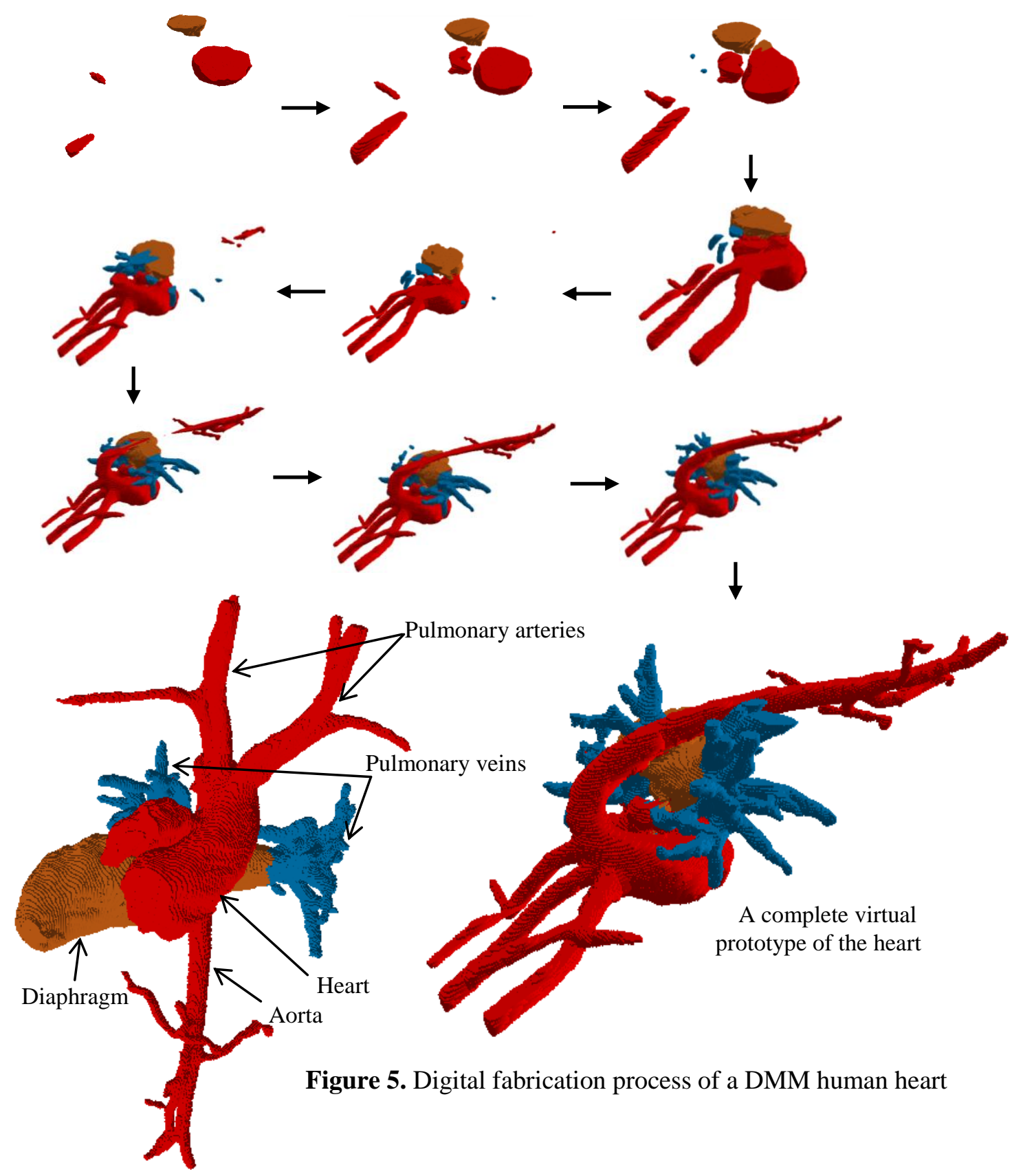


After fabrication, the resulting DMM heart prototype can be manipulated in a full-immersive VR environment, as shown in figure 6 , to visualize the quality of the prototype that the MMLM machine will subsequently deliver. A build-time estimation algorithm has been developed to estimate the fabrication time [30]. Besides, dimensional deviations of the prototype beyond a tolerance limit can be identified by superimposing the colour heart STL model on its digital prototype. As such, biomedical engineers can conveniently perform design iterations to improve the surface texture of the resulting prototype. Thus, an optimal combination of process parameters, such as layer thickness, build direction, and hatch space can be obtained for cost effective fabrication of physical biomedical prototypes.

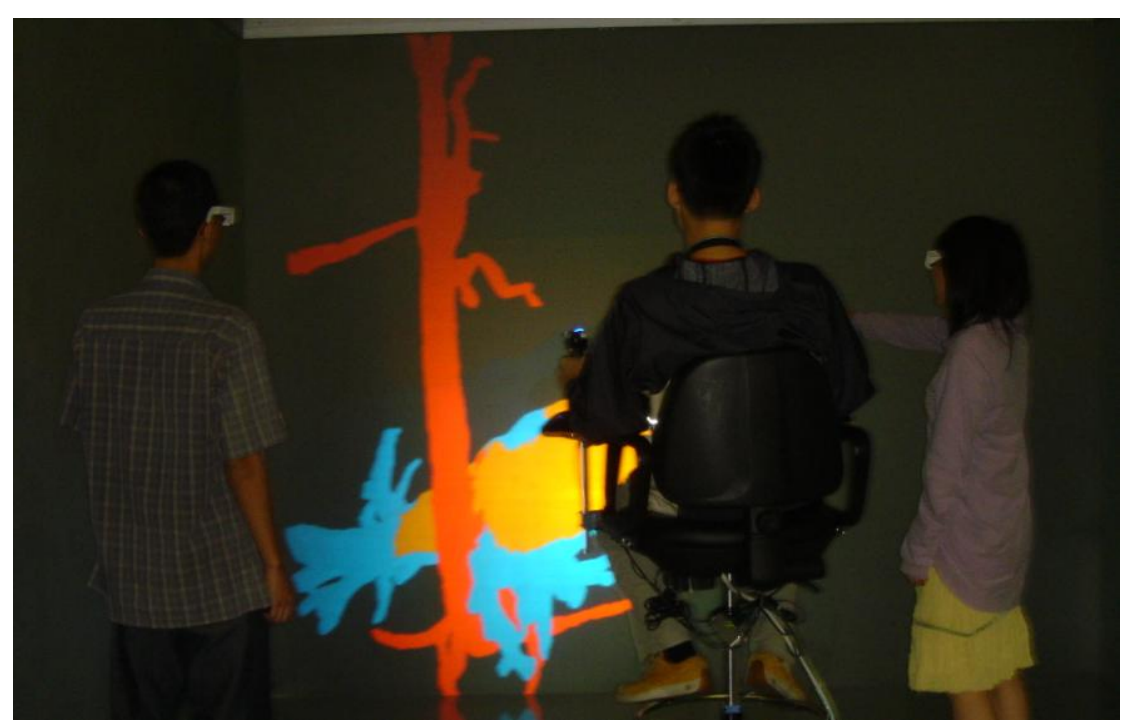

Figure 6. Visualization and analysis of the heart prototype in full-immersive VR environment

\subsection{The FGMVP module}

Some biomedical applications may require prototypes made of functionally graded materials to mimic biological and mechanical characteristics of the organs being repaired or replaced. To achieve this, the system is enhanced to represent and fabricate FGM objects. The following section describes the FGMVP module for modelling and digital fabrication of FGM objects in detail.

The FGMVP module is characterized by a contour-based FGM modeller, in which an FGM object is represented by material control functions and discretisation of layer contours with topological hierarchy. Material control functions are specified across contour families of some representative layers in the $\mathrm{X}-\mathrm{Y}$ plane and across layers along the Z-axis. The material composition at any location is calculated from control functions, and the slice contours are discretised into sub-regions of constant material composition. The discretisation resolution 
can be varied to suit display and fabrication requirements. The flow of the proposed approach is shown in figure 7.

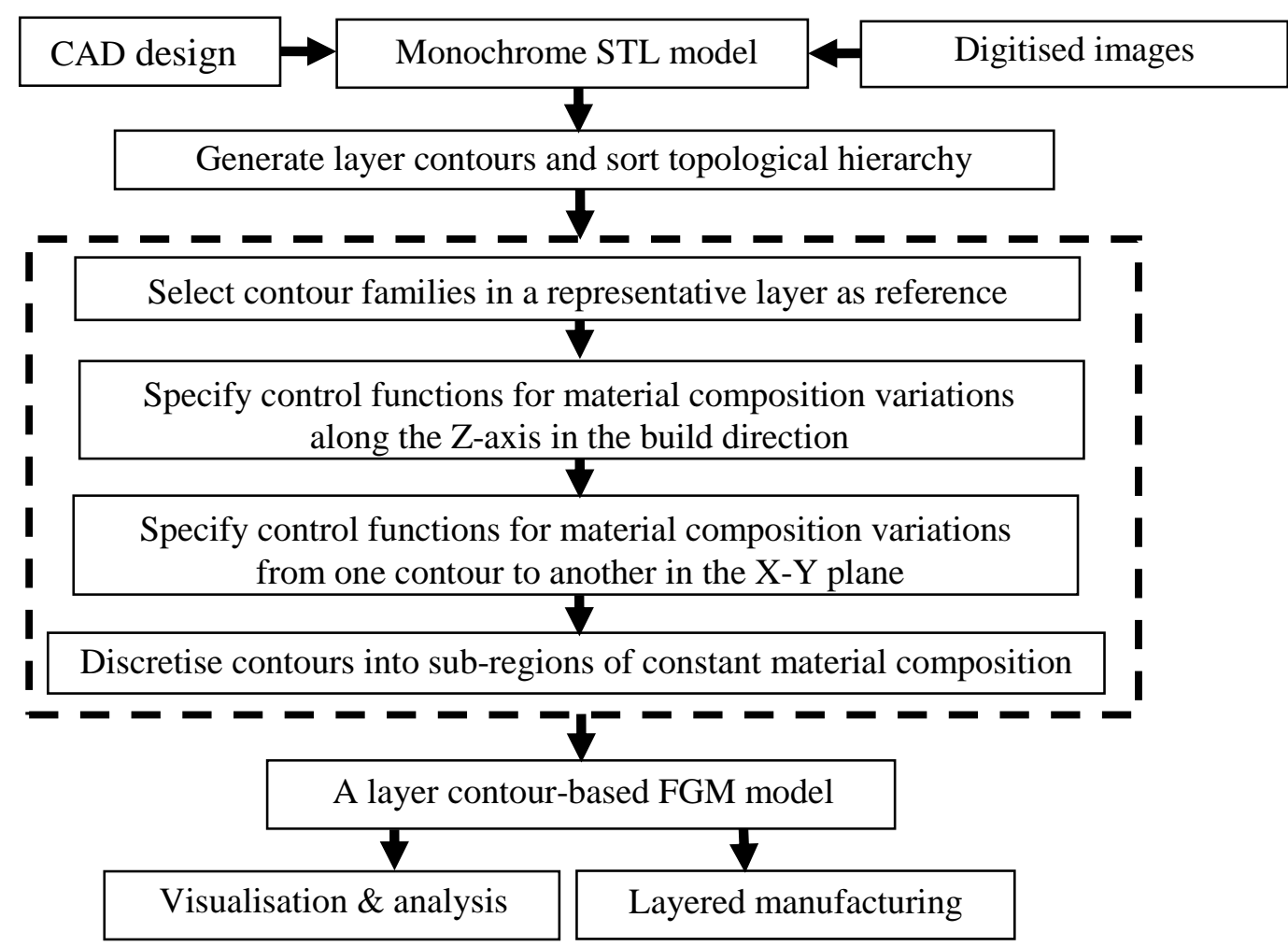

Figure 7. The flow of processing FGM objects

Firstly, the FGMVP module slices a monochrome STL model obtained from a traditional CAD design or digitized images, and sorts the resulting contours to build explicit topological hierarchy information.

Secondly, the contours are loaded into the FGMVP module for FGM object representation, with the following steps: (1) select a number of feature contour families in a representative layer; (2) specify control functions for material variations across layers along the Z-axis in the build direction; (3) specify control functions for material variations in the X-Y plane; and (4) discretise the slice contours into sub-regions of constant material composition.

Thirdly, the resulting contour-based FGM model containing both geometric and material composition variation information is processed for visualization, analysis, and fabrication of FGM objects.

In comparison with voxel-based representation schemes, this approach is computationally efficient and requires little memory for processing relatively complex objects. More importantly, it may be adapted to facilitate physical fabrication on MMLM machines when 
available. The detail of the contour-based FGM modeller was presented in [31, 32]. In the following sections, a hip joint is processed to illustrate the use of the FGMVP module for modelling and digital fabrication of FGM biomedical objects.

Figure 8 shows an artificial hip-joint assembly [33]. To fabricate such a joint, the FGM components have to be represented accordingly. Figure 9 shows a monochrome STL hip-joint assembly model with two components, namely a stem and a femoral ball head. For better medical properties, the stem should have a biocompatible material at the outer surface and a mechanically tough material at the core. Similarly, the femoral ball head should have a wearresistant outer surface and a mechanically tough core.

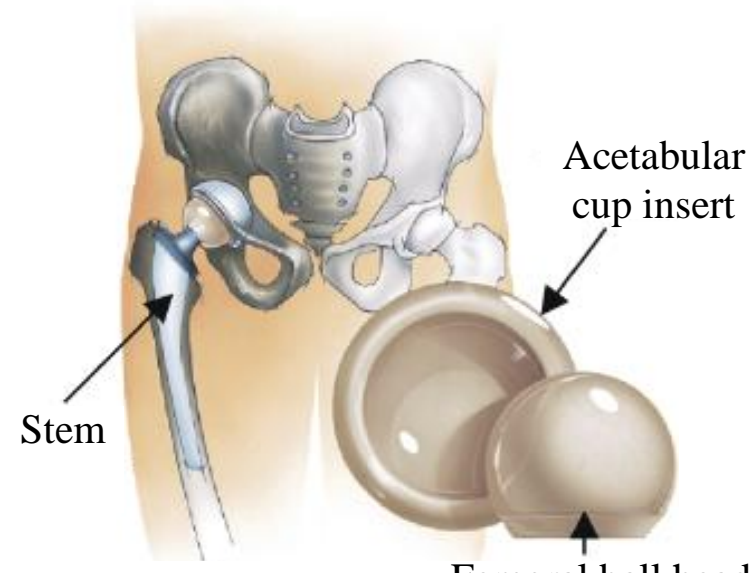

Figure 8. An artificial joint for hip prosthesis [33]

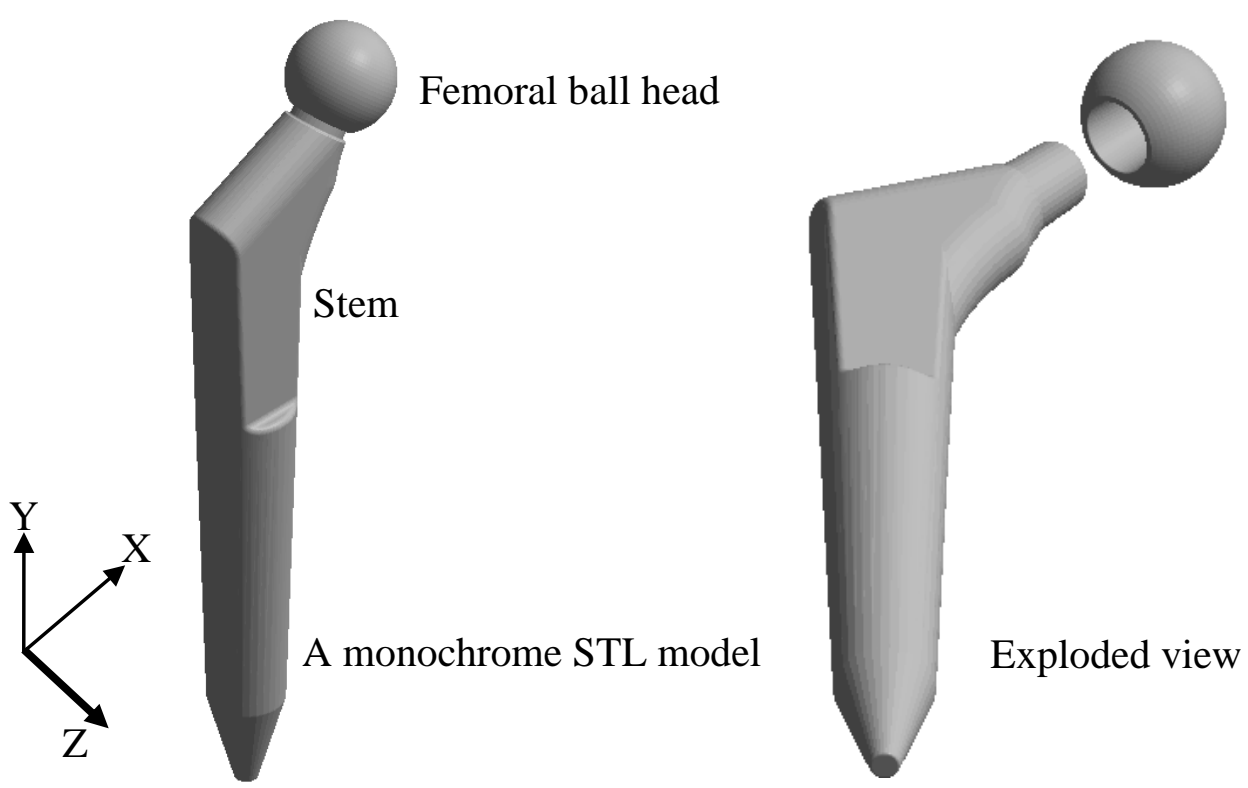

Figure 9. Prosthesis assembly with a femoral ball head and a stem for hip joint replacement 
Using the FGMVP module, the STL hip-joint assembly model is firstly sliced into a set of layers as a homogeneous contour-based model as in figure 10, and the topological hierarchy information of each layer is established; secondly, a feature layer is selected for assigning primary materials and material control functions for calculation of material composition; thirdly, each layer is discretised into sub-regions of constant material composition. Subsequently, the resulting geometric contours and material information are used for visualization and digital fabrication of the FGM hip-joint assembly model.
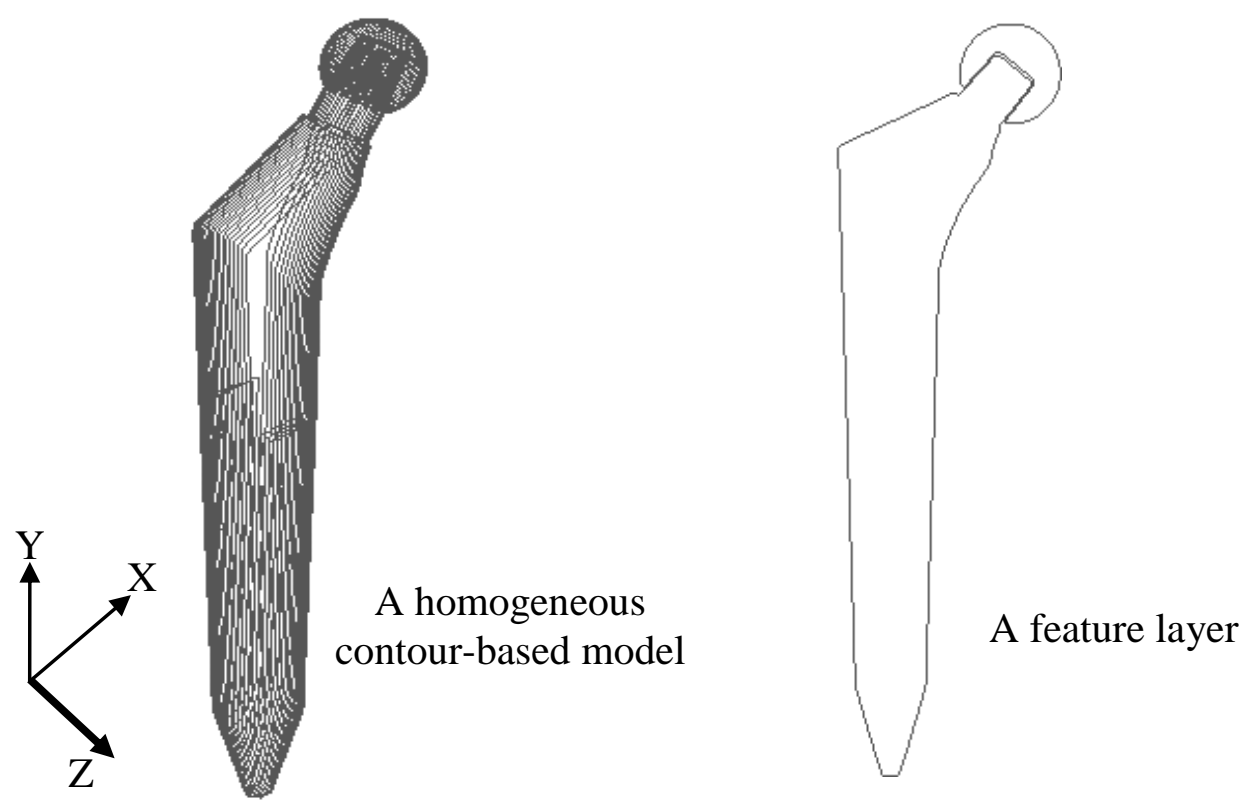

Figure 10. A homogeneous contour-based hip-joint assembly and a feature layer for assigning primary materials and material control functions

Figure 11 shows the resulting FGM layers in a wireframe and a rendered display mode, respectively. For the stem, it has a yellow/black graded variation in $\mathrm{X}-\mathrm{Y}$ to represent a gradual change of material composition from hydroxyapatite at the surface to titanium at the core, giving the desirable biocompatible properties at the surface and the desirable mechanical properties at the core. For the femoral ball head, it has a blue/black graded variation in X-Y plane to represent a gradual change of material composition from an alumina at the surface to a zirconia at the core, providing a wear-resistant outer surface and a tough core. Moreover, the discretisation resolution can be easily changed accordingly to control the smoothness of material composition variations. Figure 12 highlights the digital fabrication process of an FGM hip-joint assembly prototype. 


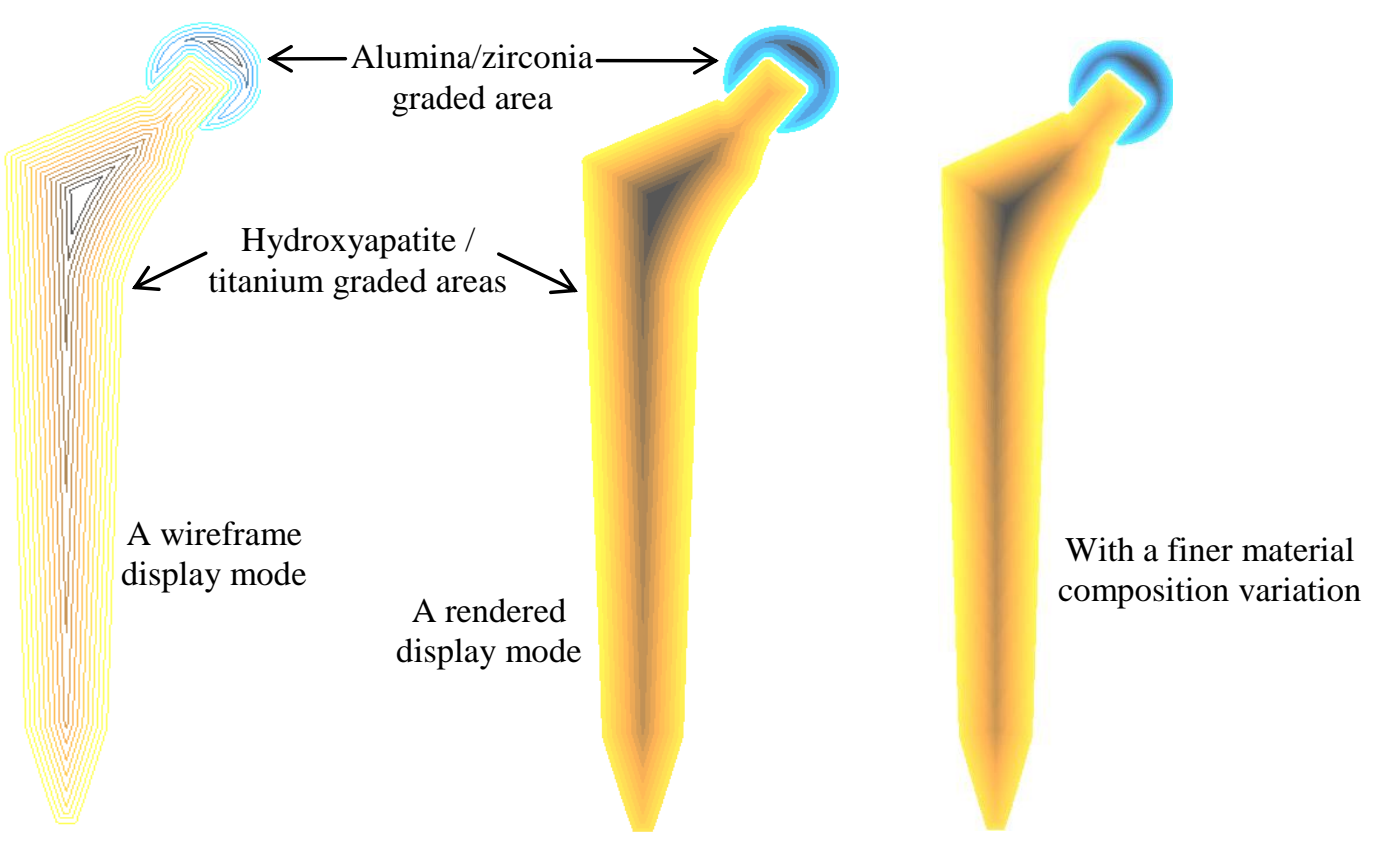

Figure 11. The resulting FMG layer of the hip-joint assembly in figure 10
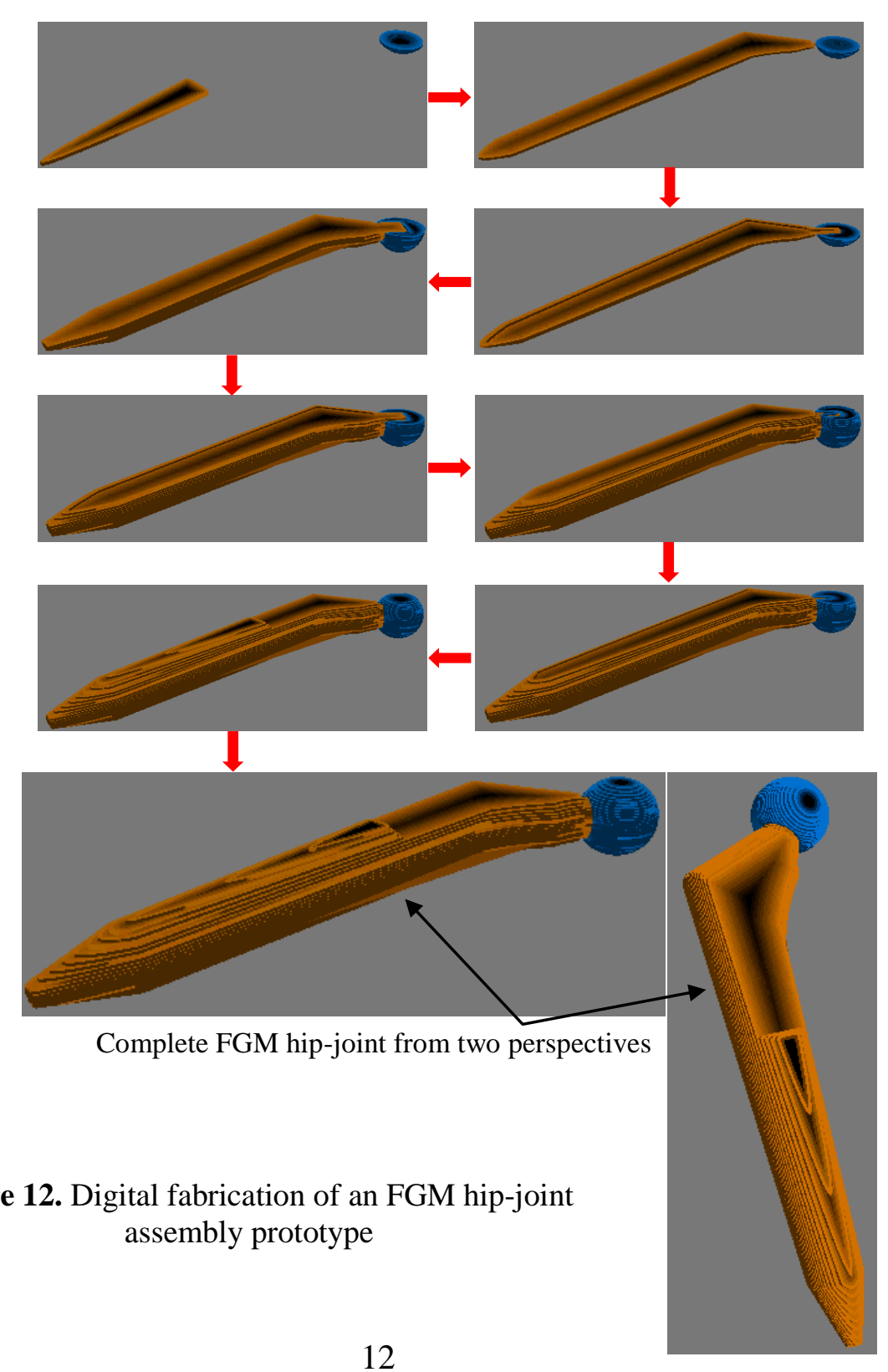

Figure 12. Digital fabrication of an FGM hip-joint assembly prototype 


\subsection{The VP system}

A virtual reality (VR) simulation module integrates the DMMVP module and the FGMVP module above to form a versatile VP system for modelling and digital fabrication of discrete and functionally graded multi-material objects for biomedical applications. The VP system provides a platform for stereoscopic visualization and analysis of digital fabrication process of multi-material objects in a VR environment [26, 28, 29]. Through simulations, design validation and modification of a biomedical product can be iterated without incurring any manufacturing and material costs of physical prototyping. Therefore, the cost and time of product development can be reduced considerably.

\section{Case Studies}

To further demonstrate possible use of the MMVP system for modelling and digital fabrication of biomedical objects to facilitate complex surgical procedures, a DMM spine model and an FGM intervertebral disc spacer are processed in the following section.
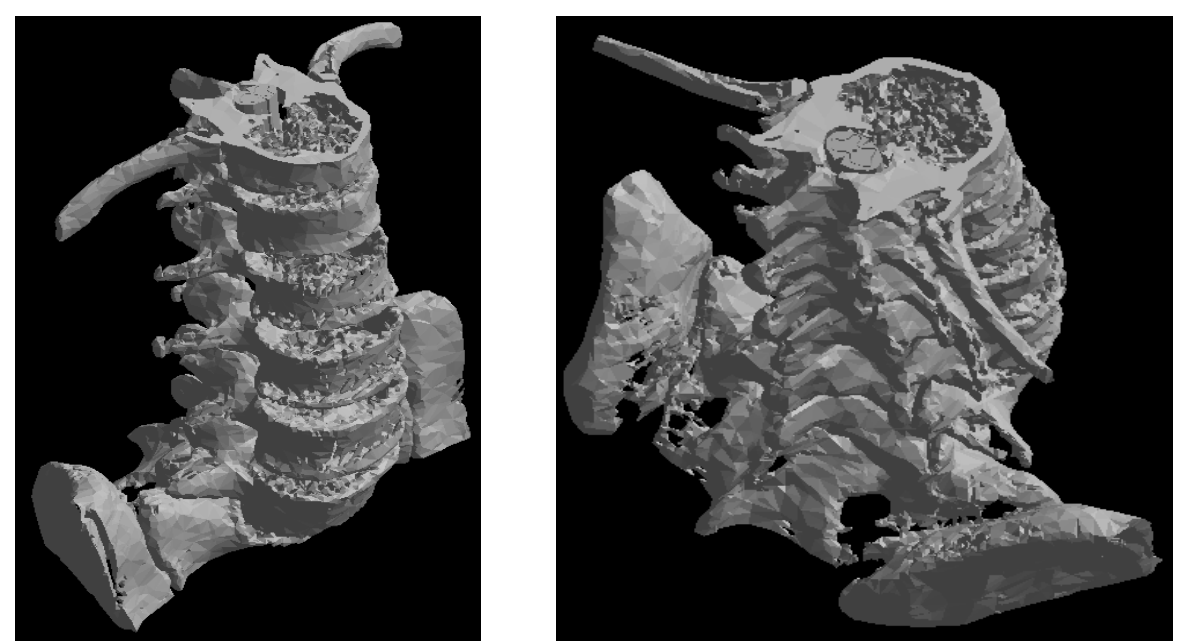

Figure 13. A monochrome STL model of a human spine

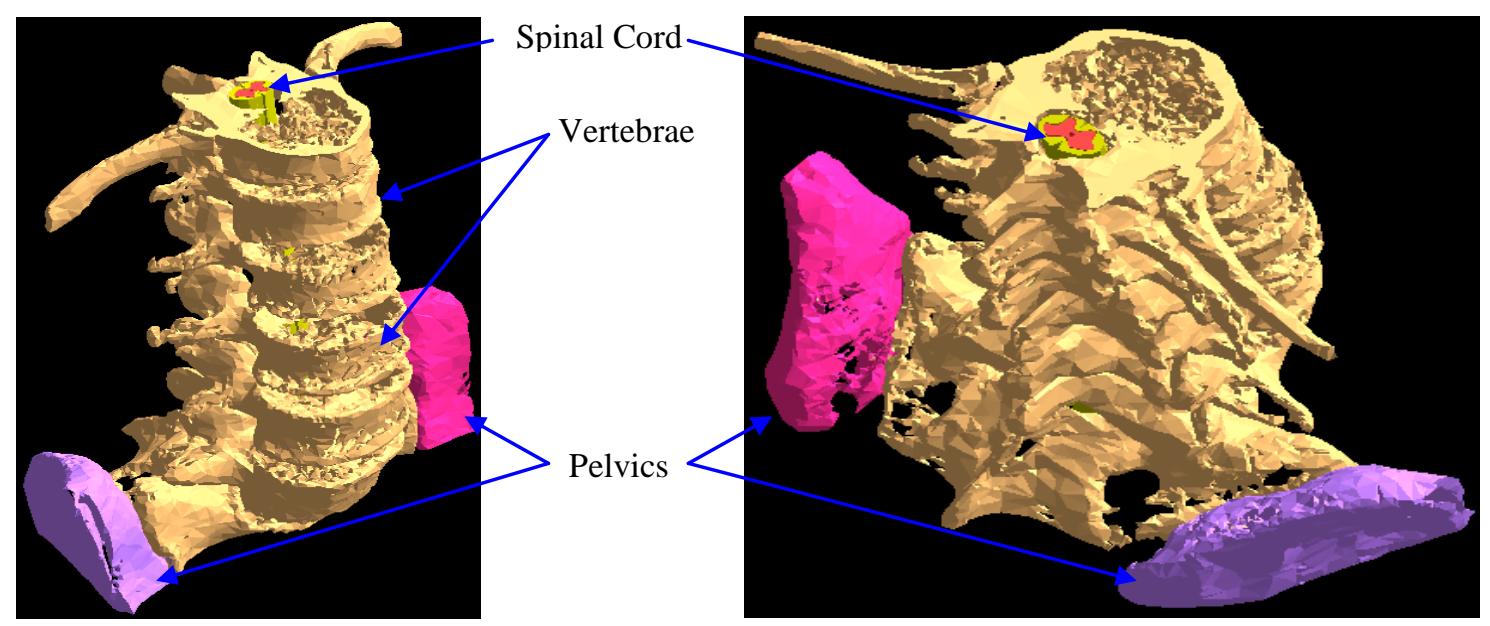

Figure 14. A colour STL model of the human spine 


\subsection{A multi-material human spine model}

Figure 13 shows a monochrome STL model of a human spine, which may not be easy to differentiate the structure of the spine. Using the proposed DMMVP module, the monochrome spine STL model is firstly painted, as shown in figure 14, to highlight various parts of the spine. As such, the spine structure can be vividly visualized and easily identified by surgeons to explain and plan complex spine surgical operations.

Based on both geometric and material information provided by the colour STL model, the DMMVP module can slice the spine model into a number layers with predefined layer thickness. The layer contours are then sorted to obtain explicit topological hierarchy information for planning and generation of concurrent toolpaths. Subsequently, a spine prototype with discrete multi-material can be digitally fabricated using the VP module. Figure 15 shows some stages of the digital fabrication process and the resulting DMM spine prototype from different perspectives.
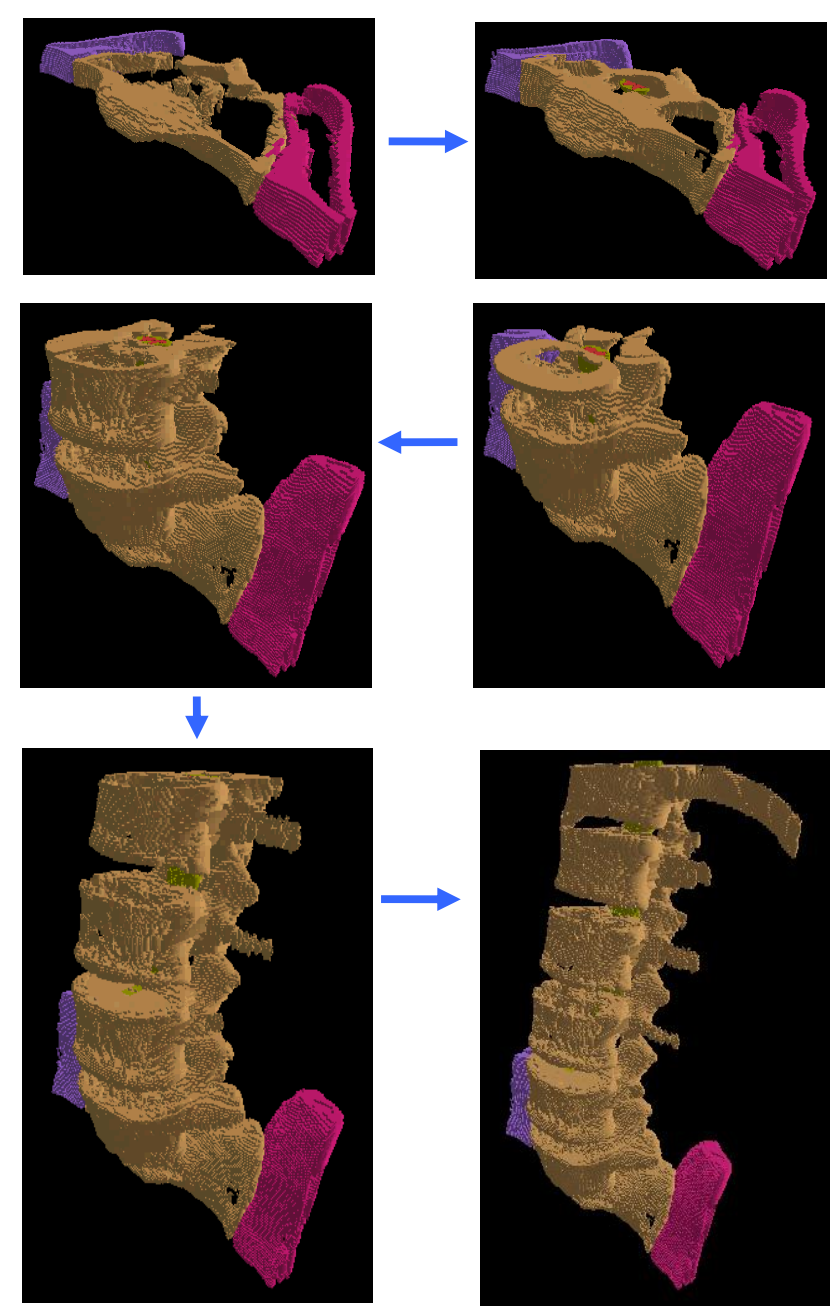

Different stages of digital fabrication process
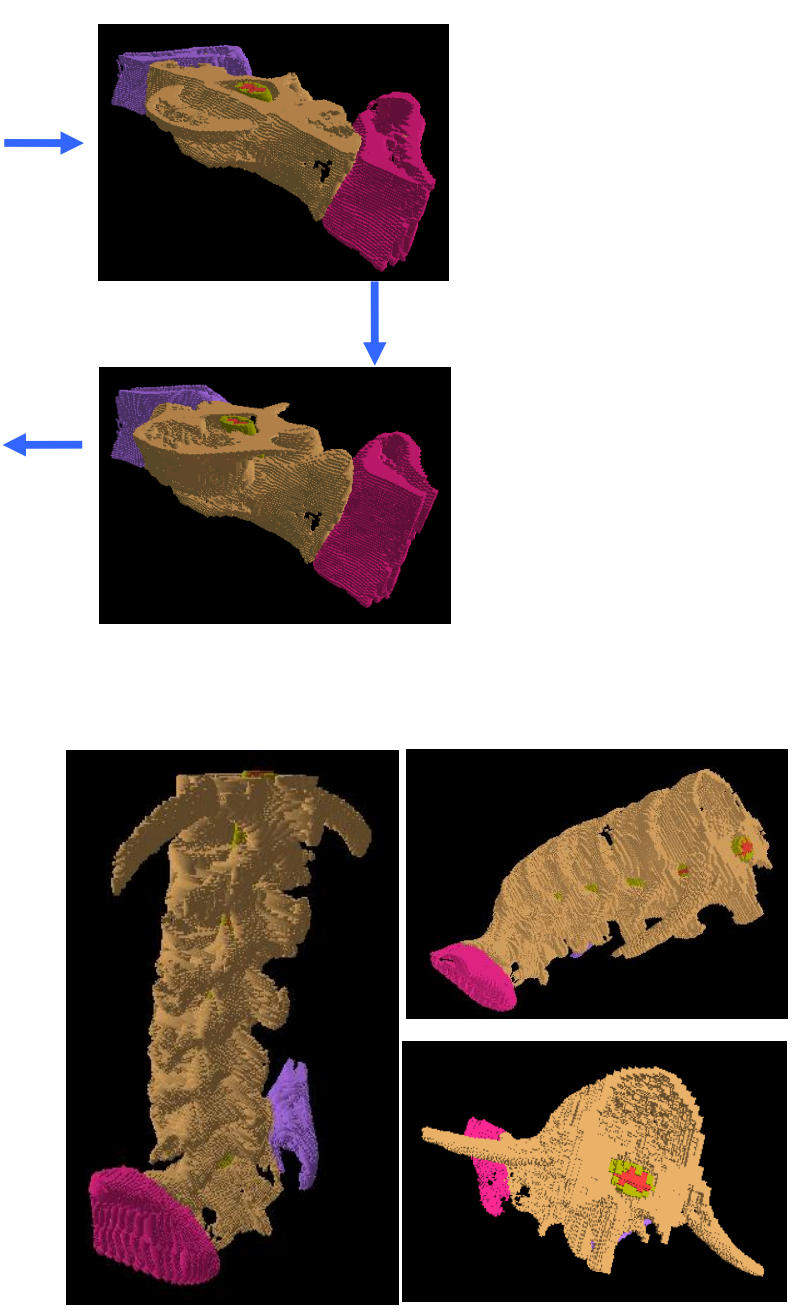

Resulting DMM spine prototype from various perspectives

Figure 15. Digital fabrication of a DMM spine prototype 
Moreover, biomedical engineers can study the quality of the resulting DMM spine prototype through visualization of the prototype and identify areas with excessive dimensional deviations. As a result, the engineers can conveniently perform design iterations to improve the surface texture of the resulting prototype. Thus, an acceptable combination of process parameters, such as layer thickness, build direction, and hatch space can be gained for cost effective fabrication of physical biomedical prototypes.

\subsection{An FGM intervertebral disc spacer}

Back pain is among the most frequently reported musculoskeletal problems. The most common factor causing low back pain is the degeneration of intervertebral discs. Taksali et al. [34] reported that back pain can result from irritation of the surrounding sinuvertebral nerves when the nucleus extrudes into annular tears. To alleviate this problem, it may be necessary to replace the degenerated disc with an artificial intervertebral disc spacer to remove the primary pain generator while preserving functional motion. For this purpose, the disc spacer should have functional and biocompatible properties compatible with a normal disc.

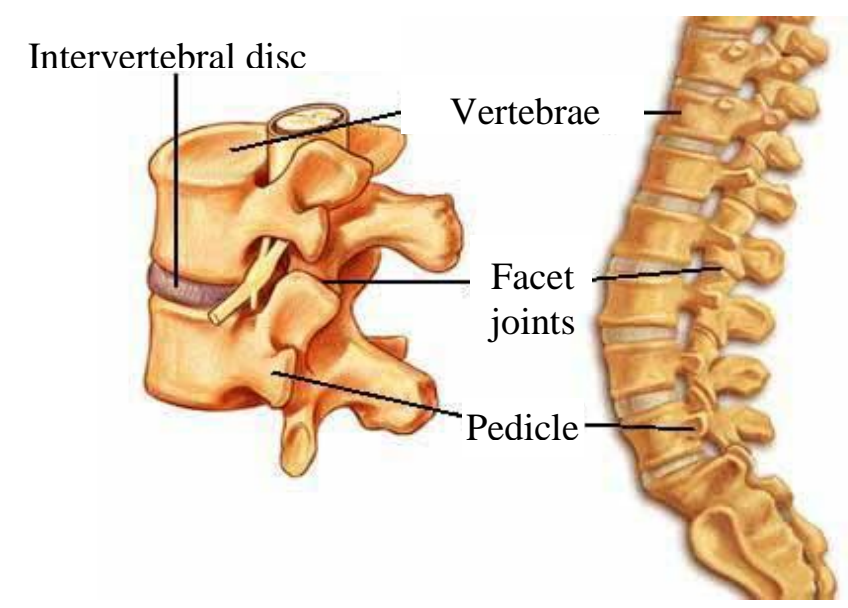

Figure 16. An intervertebral disc to be located between two adjacent vertebras [35]

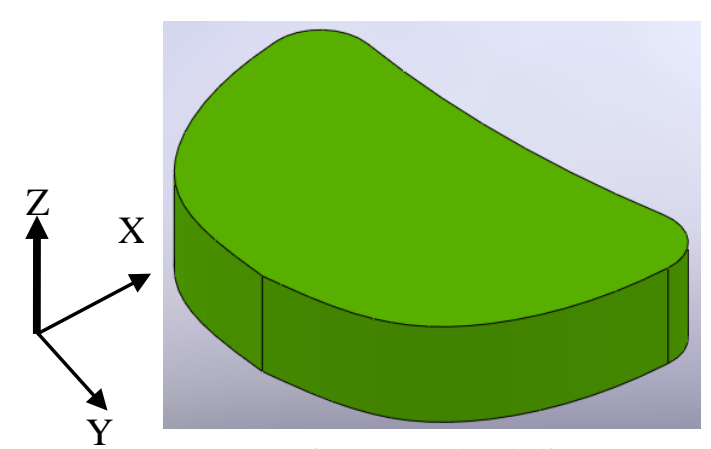

An intervertebral disc

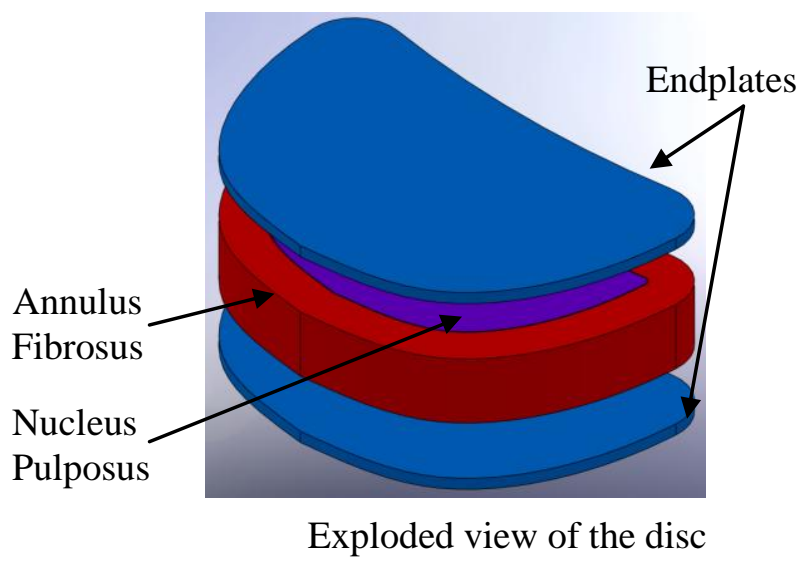

Exploded view of the disc

Figure 17. The structure of an intervertebral disc 
Figure 16 shows an intervertebral disc located between two adjacent vertebras [35]. The intervertebral disc is composed of three components, namely the nucleus pulpousus, the annulus fibrosus, and the vertebral endplates, as shown in figure 17. In comparison with the material property of the annulus fibrosus, the nucleus pulpousus is softer while the endplates are harder. Parsons et al. [36] invented a moulding method to fabricate a functional and biocompatible intervertebral disc spacer. The disc spacer consisted of (1) a central core of a biocompatible elastomer with a hardness in the range of 20-70 shore-A, shaped and sized to approximate the nucleus pulposus of a natural intervertebral disc, and (2) an outer ring of elastomeric material with a hardness in range of 40-80 shore-A, surrounding the central core to approximate the size and shape of a natural annulus fibrous. The endplates comprised a biocompatible elastomer with a hardness in range of 90-100 shore-A, and affixed, one to each end, to the outer ring / central core. The relative size of the central core and the thickness of the outer ring in the radial direction, and the selection of material hardness, could be varied in order to better match the mechanical properties of the composite prosthesis with that of the normal disc. The disc spacer can thus achieve the desired properties by varying the hardness of the elastomeric material along the circumferential and the Z-axis directions of the spacer, respectively, by changing the composition of the elastomeric material. It is therefore desirable to design and fabricate an FGM intervertebral disc spacer with a gradual change of compositions of the elastomeric material to achieve the desired hardness properties in order to mimic the normal disc in an intervertebral disc prothesis.

The FGMVP module may be a practical tool to model an FGM intervertebral disc spacer with a functional gradation in hardness property and then build a digital prototype to facilitate visualisation and subsequent fabrication. The following section describes the details.

Figure 18 shows an artificial intervertebral disc spacer inserted between two adjacent vertebrae of a human spine. To achieve the desired properties, the material composition of the disc spacer along the Z-axis is varied parabolically to form two hard ends of 100 shore-A elastomer at the extreme layers, and a soft midsection of 60 shore-A elastomer at the middle layer.

There is also a gradual variation in the elastomer hardness across the X-Y plane of each layer to form a hard exterior and a soft core. The composition at the outermost region of each layer is determined by the material variation along the Z-axis, but it changes gradually to form a soft 20 shore-A elastomer core in the innermost region. Hence, for the first and the bottom layers, the elastomer hardness across the $\mathrm{X}-\mathrm{Y}$ plane changes gradually from 100 shore-A at the outermost region to 20 shore-A at the innermost region. Similarly for the middle layer, the 
elastomer hardness changes from 60 shore-A to 20 shore-A. This results in a disc spacer with 3D FGM.

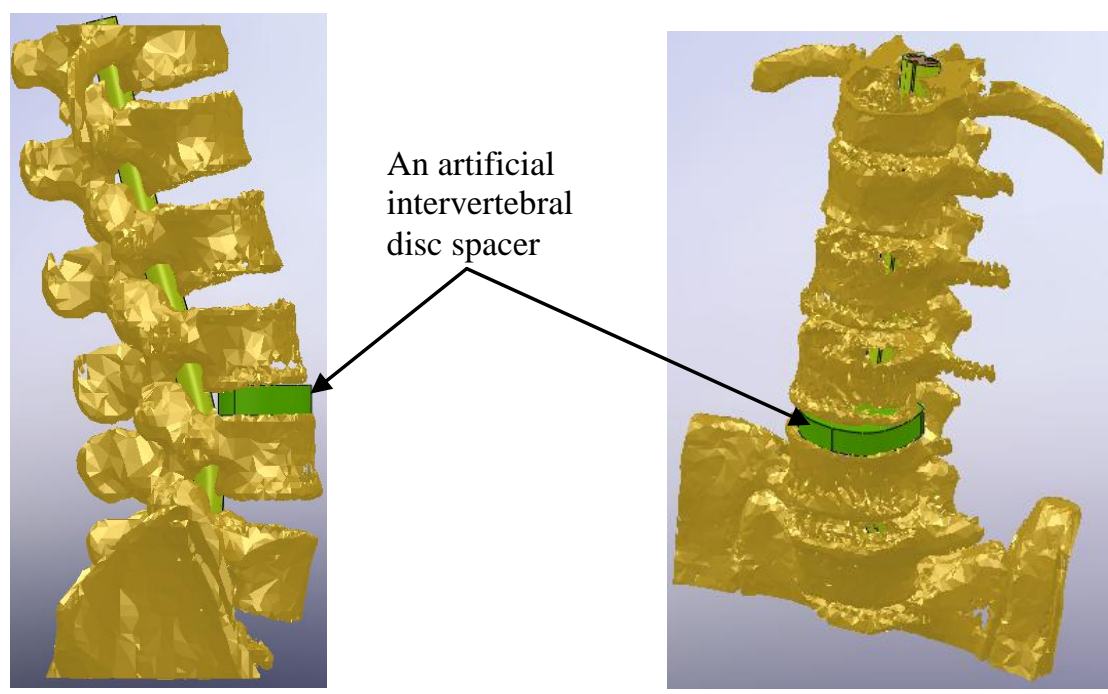

Figure 18. An artificial intervertebral disc spacer inserted between two adjacent vertebrae of a human spine

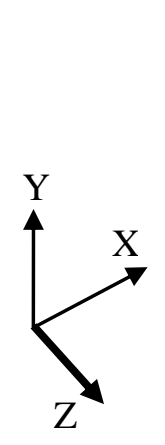

A monochrome STL intervertebral disc spacer model

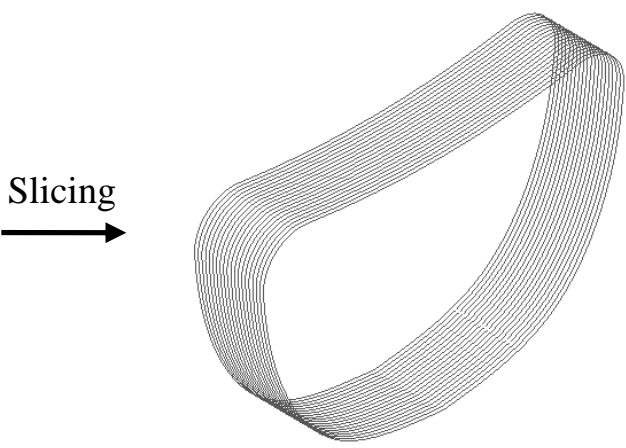

A homogeneous layer contour-based intervertebral disc spacer model

Figure 19. Slicing of the intervertebral disc spacer

To represent the material variations of the intervertebral disc spacer, it is firstly sliced into a set of homogeneous layer contour-based model of 20 layers as shown in figure 19, and explicit topological hierarchy information is then built for each layer. Secondly, feature layers are selected for assigning primary materials and material control functions for calculation of property values of material composition. Thirdly, each layer is discretised into sub-regions of constant material composition.

Figure 20 shows the resulting FGM intervertebral disc spacer with 3D variations of material composition. Along the Z-axis, it has a material variation represented by blending of blue (100 shore-A elastomer) and red (60 shore-A elastomer) colours; at the same time, there is material variation of each layer in the X-Y plane. Figure 21 shows the first layer with material variation represented by blending of blue (100 shore-A elastomer) and purple (20 
shore-A elastomer) colours in both wireframe and rendered modes, while figure 22 shows material variation in the $\mathrm{X}-\mathrm{Y}$ plane across a number of layers.

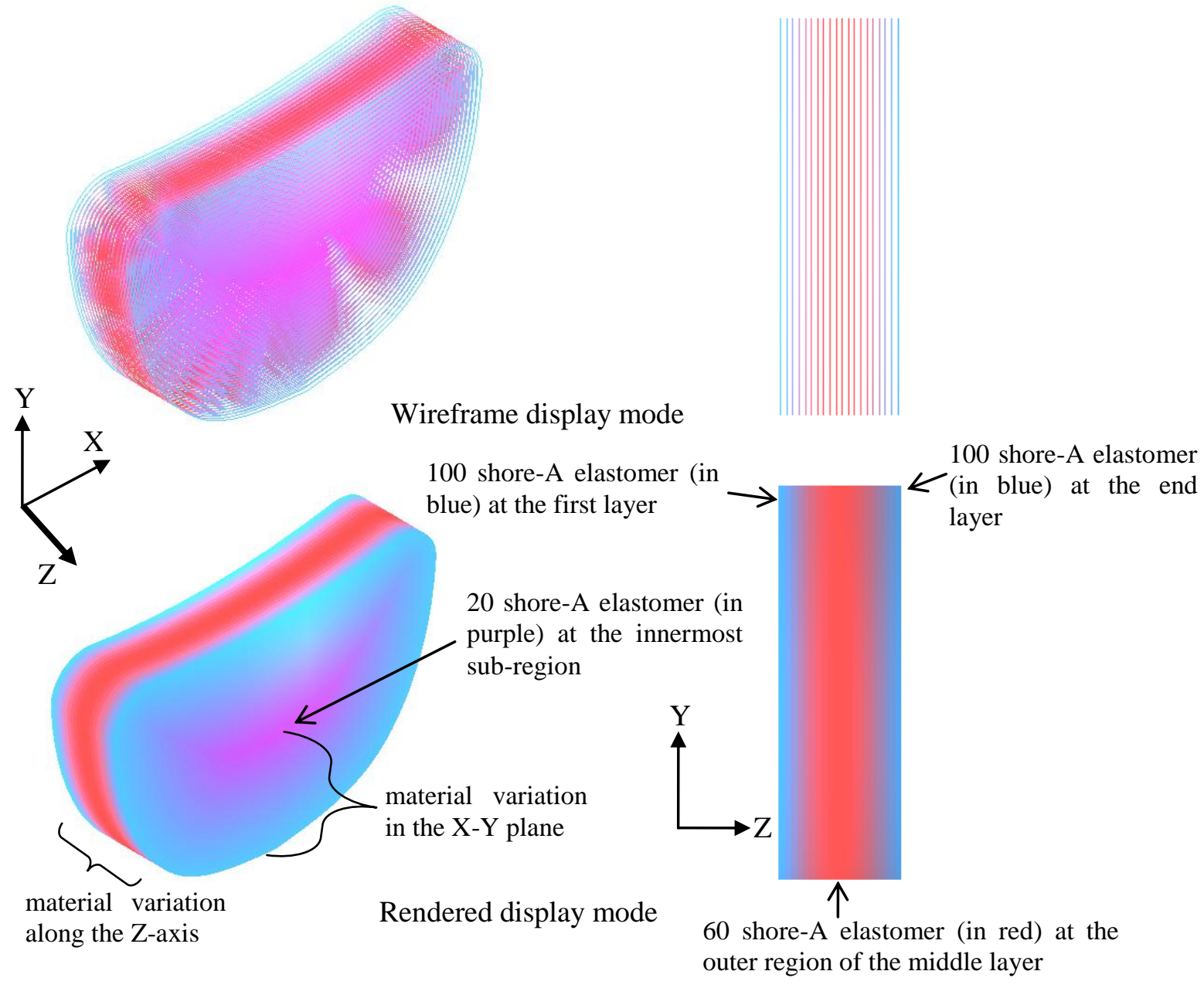

Figure 20. A contour-based FGM intervertebral disc spacer with 3D variations of material composition along the $\mathrm{Z}$-axis and in the $\mathrm{X}$-Y plane

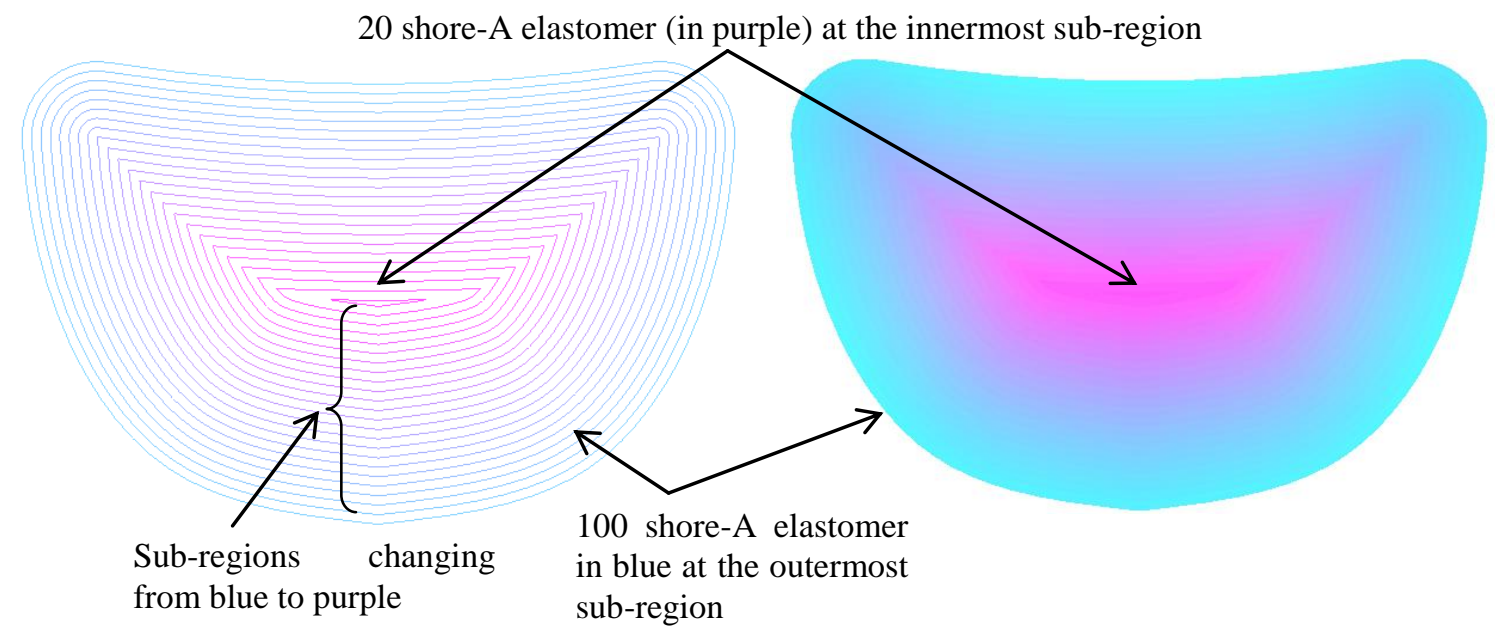

Figure 21. A layer with gradual material variation in the $X-Y$ plane 


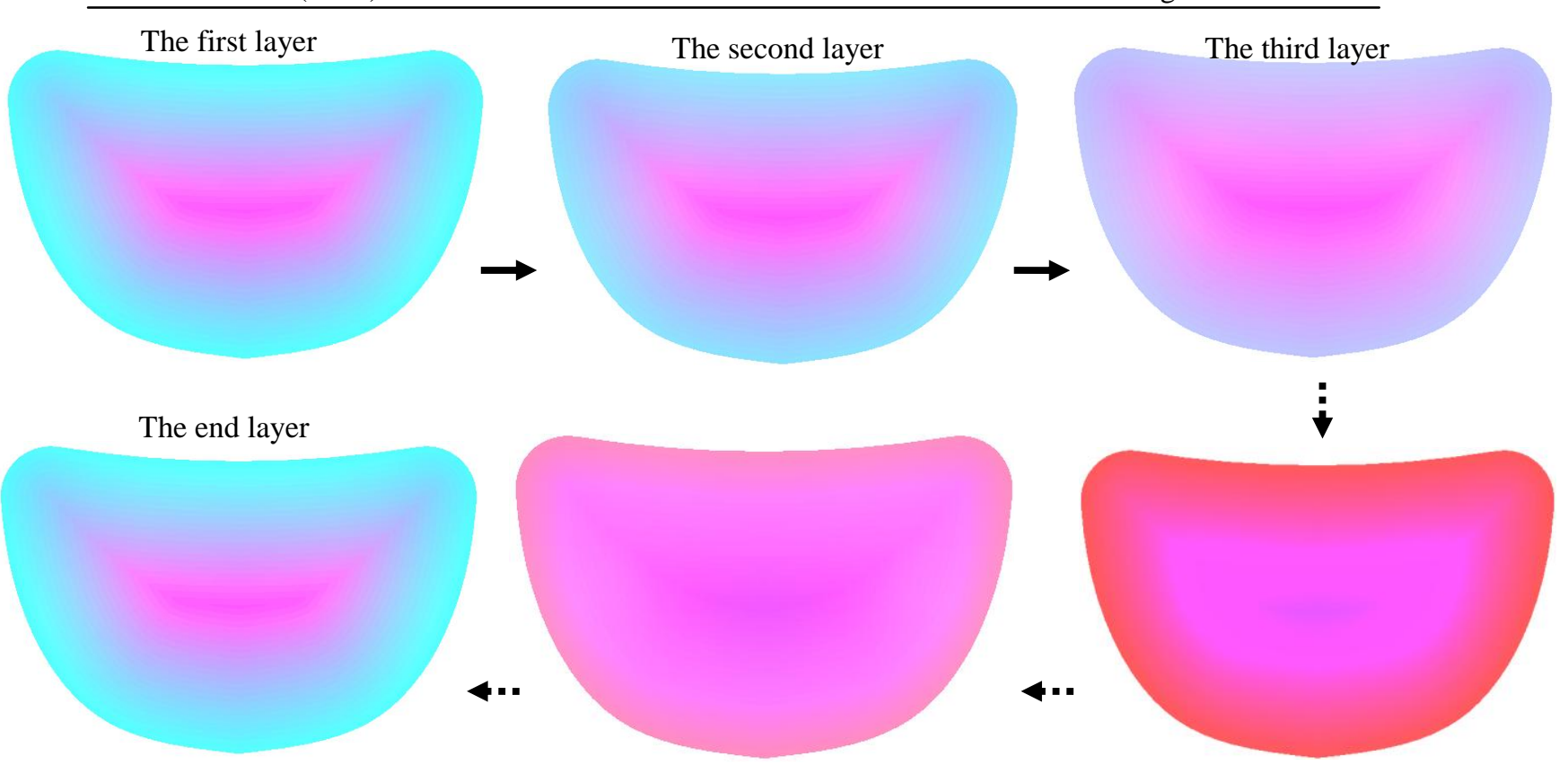

Figure 22. Material composition changes across layers
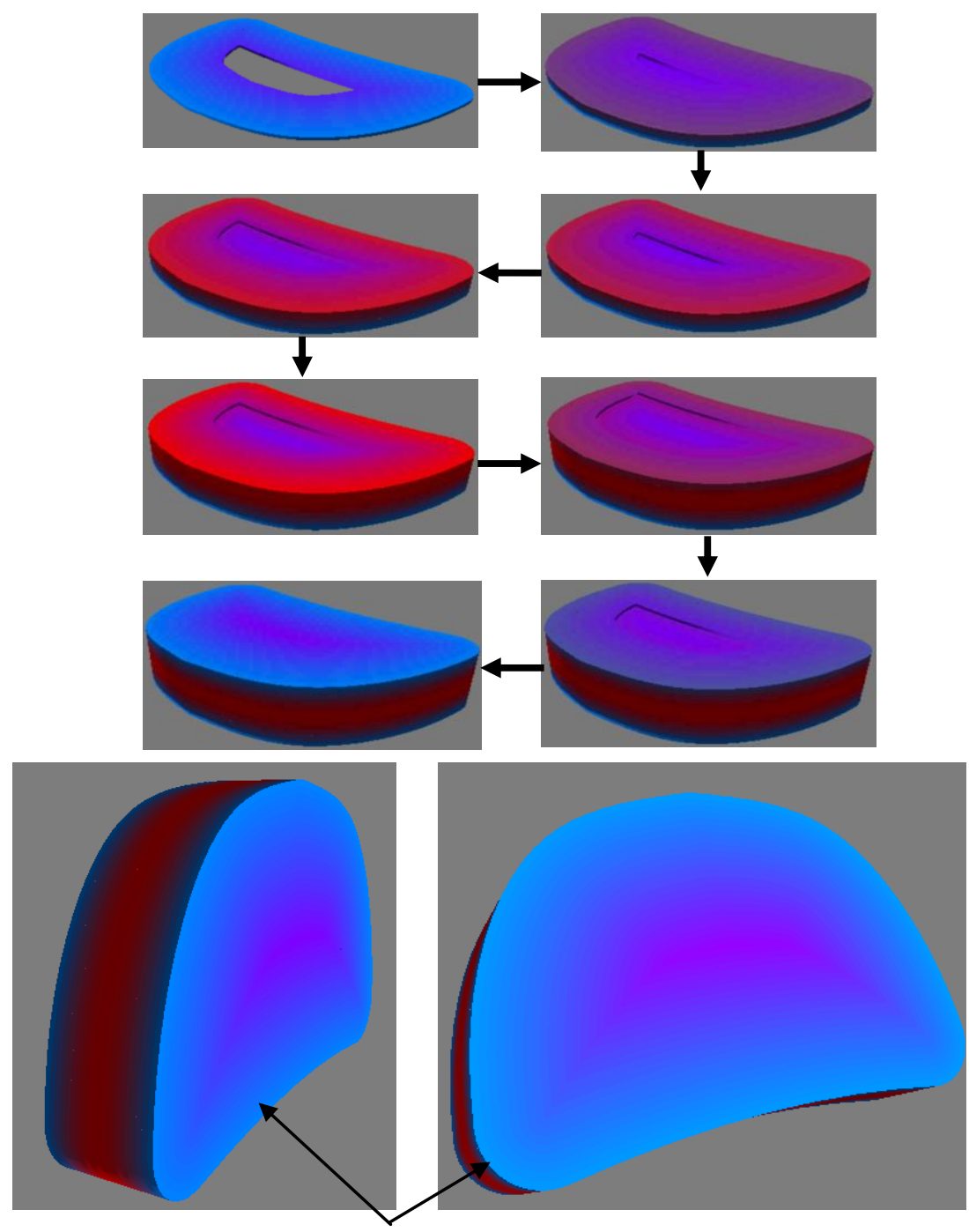

Completed FGM prototype from two perspectives

Figure 23. Digital fabrication of an FGM intervertebral disc spacer prototype 
Now, the resulting contour-based FGM intervertebral disc spacer model has both geometric and material information which can be conveniently processed for visualization of internal material variation of each layer, multi-toolpath planning, and simulation of MMLM process. Figure 23 shows the digital fabrication process of an FGM intervertebral disc spacer prototype. The FGMVP system allows adjustment of the resolution of material composition to suit practical visualization and fabrication requirements, simply by changing the discretisation of layer contours, which is the number of layers and the number of sub-regions. Therefore, it is a practical tool for modelling and digital fabrication of biomedical objects with FGM and discrete materials.

\section{Conclusion}

This paper presents a multi-material virtual prototyping (MMVP) system for modelling and digital fabrication of discrete and functionally graded multi-material objects for biomedical applications. The MMVP system is characterised by its topological hierarchy-based toolpath planning algorithm and contour-based approach to representation of FGM objects. Case studies showed that the system can conveniently process CAD models and CT/MRI images to represent complex multi-material objects for biomedical applications. It is computationally efficient and requires relatively little memory for processing complex objects. More importantly, it facilitates visualization of the resulting multi-material objects in a VR environment. The system may be adapted to control MMLM machines with appropriate hardware for physical fabrication of biomedical objects.

\section{Acknowledgements}

The authors would like to acknowledge the Research Grant Council of the Hong Kong SAR Government and the CRCG of the University of Hong Kong for their financial support for this project.

\section{References}

[1] Kumar V 1999 Solid modeling and algorithms for heterogeneous objects, Ph.D. Thesis The University of Michigan USA

[2] Shin K H, Dutta D 2001 Constructive representation of heterogeneous objects Journal of Computing and Information Science in Engineering 1 205-217

[3] Watari F, Yokoyama A, Saso F, Uo M and Kawasaki T 1997 Fabrication and properties of functionally graded dental implant Composites Part B 28B 5-11

[4] Wang G F, Shor L, Darling A, Khalil S, Sun W, Güçeri S and Lau A 2004 Precision

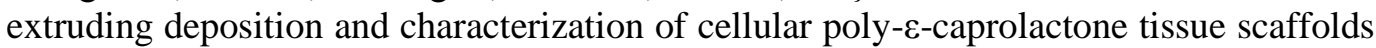
Rapid Prototyping Journal 10 420-429 
[5] Kai H, Wang S L, Madhukar K S, Qin L, Yan Y N, Zhang R J and Wang X H 2009 Fabrication of a two-level tumor bone repair biomaterial based on a rapid prototyping technique Biofabrication 2 1-7

[6] Jepson J, Beaman J J, Bourell D L and Wood K L 1997 SLS processing of functionally gradient materials, In: Bourell D L, et al. (Eds.), Solid Freeform Fabrication Symposium, Austin, Texas, The University of Texas pp. 1-8

[7] Lappo K, Jackson B, Wood K, Bourell D L and Beaman J J 2003 Discrete multiple material selective laser sintering $\left(\mathrm{M}^{2} \mathrm{SLS}\right)$ : experimental study of part processing, In: Bourell D L, et al. (Eds.), Solid Freeform Fabrication Symposium, Austin, Texas, The University of Texas pp. 109-119

[8] Merz R, Prinz F B, Ramaswami K, Terk M and Weiss L E 1994 Shape deposition manufacturing, In: Bourell D L, et al. (Eds.), Solid Freeform Fabrication Symposium, Austin, Texas, The University of Texas pp. 1-8

[9] Fessler J R, Nickel A H, Link G R, Prinz F B and Fussell P 1997 Functional gradient metallic prototypes through shape deposition manufacturing, In: Bourell $\mathrm{D}$ L, et al. (Eds.), Solid Freeform Fabrication Symposium, Austin, Texas, The University of Texas pp. 521-528

[10] Jafari M A, Han W, Mohammadi F, Safari A, Danforth S C and Langrana N 2000 A novel system for fused deposition of advanced multiple ceramics Rapid Prototyping Journal 6 161-175

[11] Jackson T R, Liu H, Patrikalakis N M, Sachs E M and Cima M J 1999 Modeling and designing functionally graded material components for fabrication with local composition control Materials \& Design 20 63-75

[12] Cho W J, Sachs E M, Patrikalakis N M and Troxel D E 2003 A dithering algorithm for local composition control with three-dimensional printing Computer-Aided Design 35 $851-867$

[13] Wang J W, Shaw L L 2006 Fabrication of functionally graded materials via inkjet color printing Journal of the American Ceramic Society 89 3285-3289

[14] Xu A P, Qu Y X, Wang J W and Shaw L L 2005 Design for solid freeform fabrication of dental restoration Proceedings of International Conference on Mechanical Engineering and Mechanics, Nanjing, China pp. 1216-1218

[15] Bandyopadhyay A, Krishna B V, Xue W C and Bose S 2009 Application of laser engineerd net shaping (LENS) to manufacture porous and functionally graded structures for load bearing implants Journal of Materials Science: Materials in Medicine Article in Press

[16] Kim H J, Chun W S and Ahn S H 2008 Fabrication of functionally graded bio materials by nao composite deposition system, In: Bártolo P J, et al. (Eds), Virtual and Rapid Manufacturing pp. 275-281

[17] Kumar V, Rajagopalan S, Cutkosky M and Dutta D 1998 Representation and processing heterogeneous objects for solid freeform fabrication The $6^{\text {th }}$ IFIP WG 5.2 International Workshop on Geometric Modeling: Fundamentals and Applications, Tokyo, Japan. The University of Tokyo

[18] Morvan S M, Fadel G M 1999 Heterogeneous solids: possible representation schemes, In: Bourell D L, et al., (Eds), Solid Freeform Fabrication Symposium, Austin, Texas, The University of Texa pp. 187-198

[19] Chiu W K, Tan S T 2000 Multiple material objects: from CAD representation to data format for rapid prototyping Computer-Aided Design 32 707-717 
[20] Hsieh C T, Langrana N A 2001 A system approach in extrusion-based multi-material CAD, In: Bourell, D.L., et al. (Eds), Solid Freeform Fabrication Symposium, Austin, Texas, The University of Texas pp, 313-321

[21] Jackson T R 2000 Analysis of functionally graded material object representation methods, Ph.D. Thesis, Massachusetts Institute of Technology, USA

[22] Shin K H 2002 Representation and process planning for layered manufacturing of heterogeneous objects, Ph.D. Thesis, The University of Michigan, USA

[23] Kou X Y, Tan S T 2007 Heterogeneous object modeling: a review Computer-Aided Design 39 284-301

[24] Samanta K, Koc B 2005 Feature-based design and material blending for free-form heterogeneous object modeling Computer-Aided Design 37 287-305

[25] Sun W, Starly B, Nam J and Darling A 2005 Bio-CAD modeling and its applications in Computer-aided tissue engineering Computer-Aided Design 37 1097-111

[26] Choi S H, Cheung H H 2005 A multi-material virtual prototyping system ComputerAided Design 37 123-136

[27] Choi S H, Cheung H H 2006 A topological hierarchy-based approach to toolpath planning for multi-material layered manufacturing Computer-Aided Design 38 143-156

[28] Choi S H, Cheung H H 2006 A CAVE-based multi-material virtual prototyping system Computer-Aided Design and Applications 3 557-566

[29] Choi S H, Cheung H H 2008 A versatile virtual prototyping system for rapid product development Computers in Industry 59 477-488

[30] Choi S H, Cheung H H 2007 Multi-material virtual prototyping for product development and biomedical engineering Computers in Industry 58 438-452

[31] Cheung H H 2007 A versatile multi-material virtual prototyping system, Ph.D. Thesis, The University of Hong Kong, Hong Kong

[32] Choi S H, Cheung H H 2009 A topological hierarchy-based approach to layered manufacturing of functionally graded multi-material objects Computers in Industry 60 349-363

[33] Anné G, Vanmeensel K, Vleugels J and Biest O V D 2005 Electrophoretic deposition as a novel near net shaping technique for functionally graded biomaterials, In: Biest O V D, et al. (Eds), The $8^{\text {th }}$ International Symposium on Multifunctional and Functionally Graded Materials (FGM 2004), Leuven, Belgium, July 11-14 pp. 213-218

[34] Taksali S M D, Jonathan N, Grauer M D, Alexander R and Vaccaro M D 2004 Material considerations for intervertebral disc replacement implants The Spine Journal 4 231S$238 \mathrm{~S}$

[35] Bridwell K M D 2009 http://www.spineuniverse.com/displayarticle.php/article1267.html

[36] Parsons J R, Lee C K, Langrana N A, Clemow A J and Chen E H 1992 Functional and biocompatible intervertebral disc spacer containing elastomeric material of varying hardness, United States Patent 